\title{
Morfologia polínica da tribo Canarieae (Burseraceae) na América do Sul ${ }^{1}$
}

\author{
CLARA ISABEL AGUILAR-SIERRA² e THEREZINHA SANT'ANNA MELHEM ${ }^{3}$
}

(recebido em 10/04/96; aceito em 10/06/97)

\begin{abstract}
Pollen morphology of the tribe Canarieae (Burseraceae) in South America). The tribe Canarieae is represented in South America by two genera, Dacryodes Valh and Trattinnickia Willd. Pollen grains from 27 collections representing 12 taxa were examined. The pollen material was acetolyzed, measured, described and photographed by light microscopy and, in most cases, also by electron microscopy scanning. The data obtained was statistically analysed by methods according to sample sizes. Dacryodes and Trattinnickia have the same intermediate size as well as form (mostly subprolate) of pollen grains. The light microscopic picture of the sexine of Dacryodes shows a psilate surface, while in Trattinnickia this is granulate; nevertheless, electron microscopic scanning examination reveals that in both taxa the surface is perforate-psilate. In the case of Dacryodes the size of pollen grains as well as the size of the apertures enable separation of D. glabra (Steyerm.) Cuatrec., D. nitens Cuatrec. and D. occidentale Cuatrec., while the other species are indistinguishable. In Trattinnickia the only characteristic that differentiated T. burserifolia Mart. from the other taxa studied was the aperture, it being brevicolpate in this species.
\end{abstract}

RESUMO - (Morfologia polínica da tribo Canarieae (Burseraceae) na América do Sul). A tribo Canarieae está representada na América do Sul por dois gêneros, Dacryodes Vahl e Trattinnickia Willd. Foram examinados os grãos de pólen de 27 exsicatas que representam 12 táxons. O material polínico foi acetolisado, medido, descrito e fotografado em microscopia óptica e, na maioria dos casos, também em microscopia eletrônica de varredura. As medidas receberam tratamento estatístico adequado ao tamanho da amostra. Dacryodes e Trattinnickia possuem características palinológicas comuns quanto ao tamanho (médio) e à forma (predominantemente subprolata). Sob microscópio óptico a sexina dos grãos de pólen de Dacryodes é psilada e em Trattinnickia parece ser granulada; não obstante, o exame ao MEV mostrou que esta é psilado-perfurada nos dois táxons. No caso de Dacryodes, o tamanho dos grãos de pólen e das aberturas permitiu separar D. glabra (Steyerm.) Cuatrec., D. nitens Cuatrec. e D. occidentale Cuatrec. sendo impossível distinguir as demais espécies; em Trattinnickia, a abertura foi o único caráter que diferenciou T. burserifolia Mart. (brevicolpada) dos outros táxons estudados.

Key words - Burseraceae, Dacryodes, Trattinnickia, pollen grains

\section{Introdução}

A tribo Canarieae é predominantemente um grupo paleotropical; está representada na América do Sul pelos gêneros Dacryodes Vahl e Trattinnickia Willd. Dacryodes desenvolve-se nas florestas chuvosas tropicais; 15 espécies distribuem-se através da Colômbia, Equador, Peru, Venezuela, Brasil e parte das Antilhas (Cuatrecasas 1957). Swart (1942) descreveu nove espécies de Trattinnickia para a zona equatorial da América, distribuídas desde o istmo do Panamá até o Peru.

Como parte da revisão das Burseraceae no Neotrópico, Daly (1989) concluiu que Trattinnickia, com flores trímeras, frutos indeiscentes com mesocarpo oleaginoso-resinoso rodeando três pirenos fundidos (no lugar do pseudo-arilo doce e suculento

1. Parte da tese de doutorado de C.I. Aguilar-Sierra, Departamento de Botânica, IB-USP.

2. Universidad Nacional de Colombia, Facultad de Ciencias, A.A. 3840 Medellín, Colombia. Bolsista da RLB e do Programa Mutis.

3. Instituto de Botânica, Caixa Postal 4005, 01061-970 São Paulo, Brasil. Bolsista do CNPq. que envolve os pirenos livres de Protieae) e pecíolos com feixes vasculares anômalos na medula (Solereder 1908), caracteres quase completamente ausentes em Protieae e predominantes em Canarieae, devia ser transferido para esta tribo. Dacryodes é um gênero de árvores não muito altas, com flores muito pequenas, cálice ligeiramente cupular, pétalas livres e patentes (ou fortemente reflexas) na antese, fruto elipsoidal ou raramente globoso, com endocarpo finamente cartilaginoso; as árvores do gênero Trattinnickia normalmente alcançam o dossel da floresta e têm flores relativamente grandes, cálice fortemente cupular, corola tubular longa com pétalas conatas em pelo menos $2 / 3$ do seu comprimento, fruto umbonado a depresso-ovóide com endocarpo pétreo, trilobado (Daly 1989).

Os estudos palinológicos da tribo Canarieae abrangem dados polínicos para espécies dos gêneros: Canarium L. em Erdtman (1952), Mitra et al. (1977), Forman et al. (1989) e Segaar \& van der Ham (1993); Dacryodes, em Mitra et al. (1977), Forman et al. (1989) e Segaar \& van der Ham (1993); Haplolobus Lam em Forman et al. (1989) e Segaar \& van der Ham (1993); Paclylobus Don em Erdtman (1952), 
Mitra et al. (1977) e Segaar \& van der Ham (1993); Rosselia Forman em Forman et al. (1994); Santiria Bl. em Mitra et al. (1977), Forman et al. (1989) e Segaar \& van der Ham (1993); Scuthinante Thw. em Segaar \& van der Ham (1993) e Trattinnickia em Roubik \& Moreno (1991).

O presente trabalho visa caracterizar morfologicamente os grãos de pólen de 12 táxons da tribo Canarieae presentes na América do Sul.

\section{Material e métodos}

O material polínico foi coletado de botões florais das seguintes exsicatas, sendo assinalada com um asterisco $(*)$ aquela selecionada como material padrão:

Dacryodes chimantensis Steyerm. \& Mag. Colômbia: Amazonas: Río Caquetá, Araracuara, H. Vester, A. Matapi 265, 26-II-1991, det. D. Daly $(\mathrm{COAH})^{*}$. D. glabra (Steyerm.) Cuatrec. Brasil: Pará: Tucuruí, F.E. Miranda et al. 402, 2-IX-1983, det. D. Daly (INPA)*. D. granatensis Cuatrec. Colômbia: Valle: Río Anchicayá, J. Cuatrecasas 14413 (Isótipo), 16-IV-1943, det. J. Cuatrecasas, conf. D. Daly $(\mathrm{COL})^{*}$. D. nitens Cuatrec. Colômbia: Valle: Buenaventura, Bajo Calima, A. Gentry 53700, det. D. Daly (CUVC)*. D. occidentale Cuatrec. Colômbia: Antioquia: San Carlos, Alto de Samaná, R. Fonnegra et al. 3043, 14-VI-1989, det. R. Callejas, conf. D. Daly (HUA); R. Fonnegra et al. 3046, 14-VI-1989, det. D. Daly (HUA)*; San Luis, Río Samaná-Río Claro, J.J. Hernández et al. 559, 11-XI-1982, det. D. Daly (HUA). Valle: Buenaventura, Bajo Calima, M. Monsalve 616, 13-XII1984, det. D. Daly (CUVC). D. peruviana (Loesn.) Lam. Brasil: Amazonas: Rio Negro, G.T. Prance et al. 16147, 05-XI-1971, det. D. Daly (INPA). Colômbia: Valle: Buenaventura, Bajo Calima, D. Daly et al. 6016, 17-V-1989, det. D. Daly (HUA)*; M. Monsalve 597, 30-XI-1984, det. D. Daly (JAUM). Equador: Napo, W. Palacios 2807, 30-VIII-1988, det. W. Palacios (JAUM). Peru: San Martín, J. Schunke 4445, 24-IX-1970, det. D. Daly (INPA). D. cf. roraimensis Cuatrec. Colômbia: Amazonas: Río Caquetá, Araracuara, H. Vester, A. Matapi 299, 4-III-1991, det. D. Daly (COAH)*. Trattinnickia aspera (Standl.) Swart. Colômbia: Antioquia: San Luis, Quebrada La Cristalina, J.G. Ramírez, D. Cárdenas 879, 21-V-1987, det. J. G. Ramírez, D. Cárdenas, conf. D. Daly (HUA); J.G. Ramírez, D. Cárdenas 1607, 24-IX-1987, det. J. G. Ramírez, D. Cárdenas, conf. D. Daly (HUA)*; San Luis, Vereda La Josefina, D. Cárdenas, J.G. Ramírez 2723, 12-IV-1990, det. D. Cárdenas (JAUM). T. burserifolia Mart. Brasil: Amazonas: Manaus, Ponta Negra, D. Coêlho, G. Freitas 1765, 26-VI1980, det. D. Daly (SP); Capoeirão; A. Ducke, 5-X-1927, det. A. Ducke (INPA). Mato Grosso: Rio Juruana, M.G. Silva, J. Maria 3330, 13-VII-1977, det. D. Daly (INPA). Pará: Serra dos Carajás, C. R.S. Sperling et al. $6158,17-\mathrm{V}-1991$, det. N. A. Rosa (MG). Rondônia: Rio Madeira, G. T. Prance et al. 5828, 10-VII-1968, det. W. Rodrigues (R). Colômbia: Amazonas: Araracuara, pista aérea, D. Restrepo s.n., det. M. Sánchez (COAH)*. Suriname: J.P. Schulze 7262, 10-VIII-1955, det. J. C. Lindeman (INPA). T cf. lawrancei Standl. var. bolivianum Swart. Brasil: Rondônia, C.A. Cid Ferreira et al. 4907, 30-VI-1984, det. D. Daly (MG)*. T. multiflora Cuatrec. Colômbia: Vaupés: Río Guainía, R.E. Schultes et al. 17950 (Isótipo), 2-XI-1952, det. J. Cuatrecasas, conf. D. Daly (COL)*. T. rhoifolia Willd. subsp. willdenowii Engl. Venezuela: Bolívar, F.J. Breteler 5055, 13-III-1966, det. F.J. Breteler (CUVC)*.

Os grãos de pólen foram preparados para estudos ao nível de microscopia óptica (Erdtman 1960) e de microscopia eletrônica de varredura - MEV (Aguilar-Sierra 1995). No material padrão foram obtidas medidas dos diâmetros polar e equatorial, em vista equatorial, e do diâmetro equatorial, em vista polar, de 25 grãos de pólen tomados ao acaso. Para as medidas dos materiais de comparação, das aberturas e da espessura da exina, foram utilizadas amostras de 10 grãos de pólen. Todas estas medidas receberam tratamento estatístico adequado ao tamanho da amostra. A variabilidade no número das aberturas é dada em porcentagem; foram considerados grãos de pólen raros, aqueles que não apareceram na amostra $(n=100)$, tomada ao acaso, para a contagem da variação no número de aberturas, mas foram registrados na população, representada pelo total de lâminas examinadas. As fotomicrografias foram obtidas num fotomicroscópio Olympus Vanox, com câmara de exposição automática incorporada e as elétron-micrografias num MEV Zeiss DSM-940.

\section{Resultados}

As espécies de Dacryodes estudadas apresentam grãos de pólen pequenos a médios; isopolares; prolato-esferoidais, subprolatos a prolatos; âmbito subtriangular a triangular ou subcircular, goniotremados; (2)-3-colporados, endoabertura lalongada; psilados. Enquanto as de Trattinnickia têm grãos de pólen médios; isopolares; subprolatos; âmbito subtriangular a triangular, goniotremados; (2)-3-(45-6)-colporados, endoabertura lalongada; granulados (tabelas $1 \mathrm{e}$ 2).

Aberturas - Brevicolpos em D. occidentale e $T$. burserifolia, longicolpos nas demais espécies, colpos largos em T. multiflora (figura 31), estreitos nas outras espécies, de difícil mensuração (tabela 3); endoabertura lalongada, elíptica (figuras 3, 8, 31 e 34), provida de costa (tabela 3 ); membrana do colpo granulada (figuras 24, 31, 33 e 35).

Registrou-se em: D. peruviana, $97 \%$ de grãos de pólen 3-colporados e 3\% de grãos de pólen 2-colporados; $D$. glabra e $D$. occidentale, raros grãos de pólen 2-colporados; $T$. cf. lawrancei var. bolivianum, $77 \%$ de grãos de pólen 3-colporados, $23 \%$ de 4-colporados e raros grãos de pólen 5-6-colporados; $T$. multiflora, $97 \%$ de grãos de pólen 3-colporados, 3\% de 2-colporados e raros 4-colporados; T. rhoifolia subsp. willdenowii, $99 \%$ de grãos de pólen 3-colporados e $1 \%$ de 2-colporados; T. aspera, raros grãos 
Tabela 1. Caracterização morfológica dos grãos de pólen de espécies de Dacryodes e Trattinnickia $(\mathrm{PE}=$ prolato-esferoidal; PR = prolata; $\mathrm{SP}=$ subprolata; $\mathrm{SC}=$ subcircular; $\mathrm{ST}=$ subtriangular; $\mathrm{T}=$ triangular $)$.

\begin{tabular}{lcccccc}
\hline Espécies & Tamanho & P/E & Forma & Âmbito & Cólporos & Figuras \\
\hline D. chimantensis & Médio & 1,13 & PE & SC & 3 & $1-4$ \\
D. glabra & Pequeno & 1,18 & SP & T & $(2)-3$ & $5-6$ \\
D. granatensis & Médio & 1,18 & SP & T & 3 & 7 \\
D. nitens & Pequeno & 1,16 & SP & T & 3 & $8-9$ \\
D. occidentale & Médio & 1,14 & SP & SC & $(2)-3$ & $10-14$ \\
D. peruviana & Médio & 1,43 & PR & T & $(2)-3$ & $15-16$ \\
D. cf. roraimensis & Médio & 1,14 & SP & ST & 3 & $17-18$ \\
T. aspera & Médio & 1,32 & SP & ST & $3-(4)$ & $19-20$ \\
T. burserifolia & Médio & 1,23 & SP & T & $(2)-3-(4)$ & $21-24$ \\
T. cf. lawrancei var. bolivianum & Médio & 1,30 & SP & T & $3-(4-5-6)$ & $25-27$ \\
T. multiflora & Médio & 1,25 & SP & T & $(2)-3-(4)$ & $28-33$ \\
T. rhoifolia subsp. willdenowii & Médio & 1,25 & SP & T & $(2)-3$ & $34-36$ \\
\hline
\end{tabular}

de pólen 4-colporados e $T$ burserifolia, raros grãos de pólen 2-colporados e 4-colporados. É importante destacar que, geralmente, os grãos de pólen 4-colporados são loxocolporados e apresentam o contorno em forma de "pera".

Exina - Sob microscópio óptico, psilada (Dacryodes) ou granulada (Trattinnickia); os grãos de pólen de $D$. glabra, D. nitens e D. occidentale, quando observados em vista polar, parecem ter uma exina esculturada (figura 10), devido às ondulações do teto entre as perfurações (figuras 6, 9 e 13); algo similar acontece nos grãos de pólen das espécies de Trattinnickia (figuras 20 e 24); a nexina divide-se em $n_{1}$ e $n_{2}$; ao nível da endoabertura é mais espessa. Convém ressaltar a dificuldade na delimitação das camadas da exina para a obtenção de medidas precisas (tabela 4). O exame ao MEV revelou que a sexina é psilada, densamente perfurada nas espécies de Dacryodes (figuras 6, 9, 13, 14, 16 e 18). Em Trattinnickia a sexina é psilado-perfurada; as perfurações em $T$. aspera são esparsas (figura 20), em T burserifolia estão muito próximas entre si (figura 24) e em T. multiflora e $T$. rhoifolia subsp. willdenowii são inconspícuas (figuras 29, 33, 35 e 36).

Nos materiais de comparação de $D$. occidentale (tabela 5) os dados obtidos para o diâmetro polar estão compreendidos dentro do intervalo de confiança do material padrão ou de sua faixa de variação; no caso de $D$. peruviana as medidas do diâmetro polar dos materiais de comparação estão dentro da faixa de variação exceto em $D$. cf. peruviana (Monsalve 597). Para as duas espécies, a maioria das medidas do diâmetro equatorial está fora destes intervalos.
Quanto à forma, para $D$. occidentale, o material de Fonnegra et al. 3043 (tabela 5) tem grãos de pólen prolato-esferoidais, enquanto no material padrão e nos outros de comparação estes são subprolatos. Em D. peruviana, o padrão tem grãos de pólen prolatos e nos materiais de comparação a forma variou de prolata-esferoidal (Palacios 2807, Schunke 4445), a subprolata (Prance et al. 16147) até prolata (Monsalve 597).

As medidas dos materiais de comparação de $T$. aspera (tabela 5) estão dentro da faixa de variação do material padrão, com exceção do diâmetro polar de T. aspera (Cárdenas \& Ramírez 2723); este material apresentou grãos de pólen com uma forma diferente, prolata. No caso de T. burserifolia, as medidas dos materiais de comparação mostram que todos os valores estão dentro dos intervalos de confiança do material padrão ou de suas faixas de variação.

\section{Discussão}

Daly (1989) redefiniu os limites genéricos das tribos Protieae e Canarieae transferindo Trattinnickia para Canarieae, com base em caracteres morfológicos e anatômicos. A localização de Trattinnickia junto com Dacryodes nesta tribo também é apoiada pelas características morfopolínicas que, de acordo com os resultados obtidos no presente estudo, são muito similares para os dois gêneros.

Das espécies de Dacryodes e Trattinnickia aqui estudadas, há na literatura dados polínicos apenas para T. aspera em Roubik \& Moreno (1991), cujos resultados concordam com os deste trabalho, exceto 

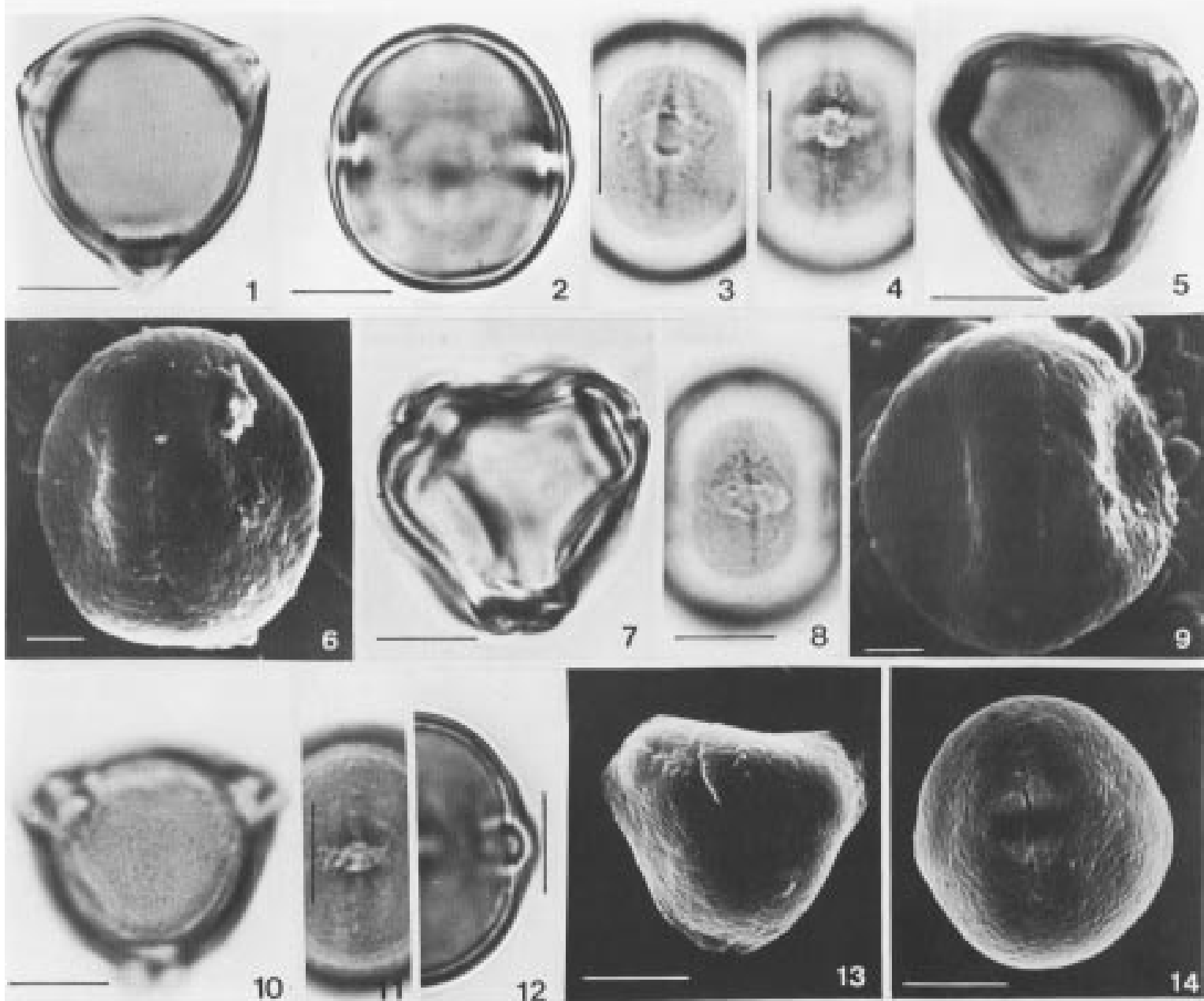

6
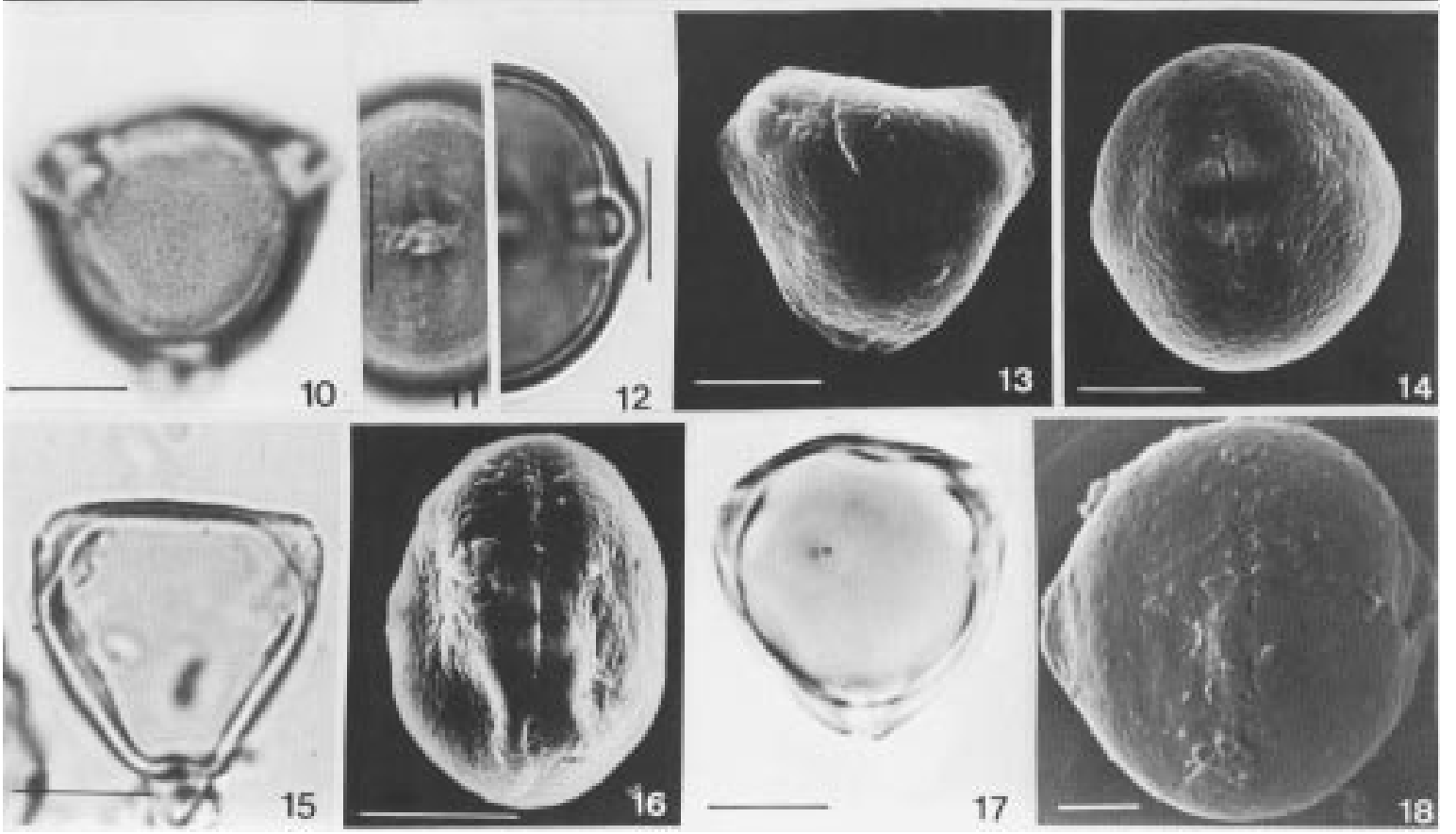

Figuras 1-18. Fotomicrografias e elétron-micrografias dos grãos de pólen de espécies de Dacryodes. 1-4. D. chimantensis. 1-2. Corte óptico, vista polar (1), vista equatorial (2); 3-4. Aberturas em diferentes níveis de focalização. 5-6. D. glabra. 5. Vista polar, contorno; 6. MEV: vista equatorial, abertura, sexina psilado-perfurada $($ escala $=4 \mu \mathrm{m}) ;$ 7. D. granatensis: vista polar. 8-9. D. nitens. 8. Detalhe do cólporo; 9. MEV: vista equatorial, abertura, sexina psilado-perfurada (escala $=4 \mu \mathrm{m}) .10-14$. D. occidentale. 10. Vista polar, superfície; 11. Detalhe do cólporo; 12. Corte óptico; 13-14. MEV. 13. Vista polar, sexina psilado-perfurada (escala $=10 \mu \mathrm{m}$ ); 14 . Vista equatorial, abertura $($ escala $=10 \mu \mathrm{m}) .15-16$. D. peruviana. 15 . Vista polar, contorno; 16 . MEV: vista equatorial, abertura, sexina psilado-perfurada $($ escala $=10 \mu \mathrm{m}) ; 17-18$. D. cf. roraimensis. 17. Vista polar, contorno; 18. MEV: vista equatorial, abertura, sexina psilado-perfurada (escala $=5 \mu \mathrm{m})$. Escala das fotomicrografias $=10 \mu \mathrm{m}$. 

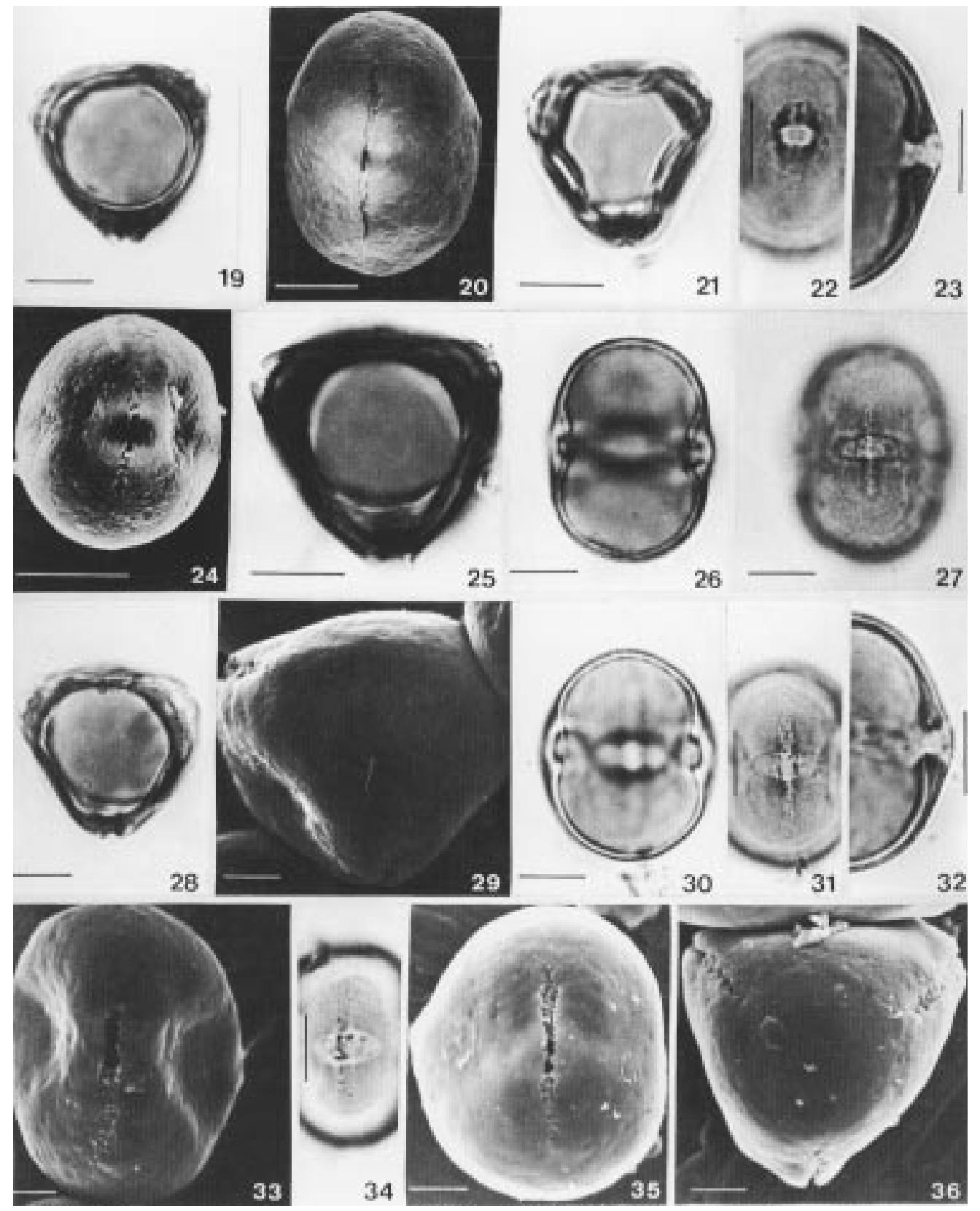

Figuras 19-36. Fotomicrografias e elétron-micrografias dos grãos de pólen de espécies de Trattinnickia. 19-20. T. espera. 19. Vista polar, contorno; 20. MEV: vista equatorial, abertura, sexina psilado-perfurada (escala $=10 \mu \mathrm{m}$ ). 21-24. T. burserifolia. 21. Vista polar, contorno; 22. Detalhe do cólporo; 23. Corte óptico; 24 . MEV: vista equatorial, abertura, sexina psilado-perfurada (escala $=10$ $\mu \mathrm{m}) ; 25-27$. Trattinnickia $\mathrm{cf}$. lawrancei var. bolivianum. 25. Vista polar, contorno; 26-27. Vista equatorial, corte óptico (26), cólporo (27); 28-33. T. multiflora. 28. Vista polar, contorno; 29. MEV: vista polar, sexina psilado-perfurada $($ escala $=4 \mu \mathrm{m})$; $30-32$. Vista equatorial, corte óptico (30), cólporo (31), detalhe da costa (32); 33. MEV: vista equatorial, abertura, sexina psilado-perfurada $($ escala $=5 \mu \mathrm{m})$; 34-36. T. rhoifolia subsp. willdenowii. 34. Vista equatorial, cólporo; 35-36. MEV. 35. Vista equatorial, abertura, sexina psilado-perfurada, membrana do colpo granulada $($ escala $=5 \mu \mathrm{m})$; 36. Vista polar, sexina psilado-perfurada $($ escala $=4 \mu \mathrm{m})$. Escala das fotomicrografias $=10 \mu \mathrm{m}$. 
Tabela 2. Medidas dos diâmetros polar e equatorial dos grãos de pólen de espécies de Dacryodes e Trattinnickia $(\mathrm{n}=25)$, em vistas equatorial (VE) e polar (VP).

\begin{tabular}{|c|c|c|c|c|c|}
\hline \multirow{2}{*}{ Espécies } & \multicolumn{5}{|c|}{ Diâmetro polar (VE) } \\
\hline & $\begin{array}{l}\text { Faixa de variação } \\
\qquad(\mu \mathrm{m})\end{array}$ & $\begin{array}{c}\overline{\mathrm{x}} \pm \mathrm{s}_{\overline{\mathrm{x}}} \\
(\mu \mathrm{m})\end{array}$ & $\begin{array}{c}\mathrm{s} \\
(\mu \mathrm{m})\end{array}$ & $\begin{array}{c}\mathrm{V} \\
(\%)\end{array}$ & $\begin{array}{l}\text { I.C. } \\
(\mu \mathrm{m})\end{array}$ \\
\hline D. chimantensis & $24,48-29,32$ & $26,49 \pm 0,22$ & 1,10 & 4,14 & $26,04-26,94$ \\
\hline D. glabra & $21,67-24,93$ & $23,43, \pm 0,19$ & 0,92 & 3,94 & $23,05-23,81$ \\
\hline D. granatensis & $25,57-35,75$ & $29,09 \pm 0,51$ & 2,57 & 8,84 & $28,03-30,15$ \\
\hline D. nitens & $21,18-25,97$ & $23,70 \pm 0,24$ & 1,19 & 5,02 & $23,21-24,20$ \\
\hline D. occidentale & $24,86-28,90$ & $26,58 \pm 0,20$ & 1,01 & 3,81 & $26,16-27,00$ \\
\hline D. peruviana & $25,88-31,09$ & $28,37 \pm 0,30$ & 1,48 & 5,22 & $27,76-28,98$ \\
\hline D. cf. roraimensis & $24,16-28,91$ & $26,99 \pm 0,28$ & 1,39 & 5,19 & $26,42-27,56$ \\
\hline T. aspera & $30,52-36,92$ & $33,44 \pm 0,34$ & 1,71 & 5,13 & $32,73-34,15$ \\
\hline T. burserifolia & $26,97-33,71$ & $29,70 \pm 0,36$ & 1,82 & 6,13 & $28,95-30,45$ \\
\hline T. cf. lawrancei var. bolivianum & $27,15-34,12$ & $30,81 \pm 0,42$ & 2,08 & 6,74 & $29,95-31,67$ \\
\hline T. multiflora & $25,48-35,11$ & $32,23 \pm 0,43$ & 2,14 & 6,65 & $31,35-33,12$ \\
\hline \multirow[t]{2}{*}{ T. rhoifolia subsp. willdenowii } & $27,33-32,67$ & $30,37 \pm 0,28$ & 1,41 & 4,63 & $29,79-30,95$ \\
\hline & \multicolumn{5}{|c|}{ Diâmetro equatorial (VE) } \\
\hline D. chimantensis & $21,58-26,38$ & $23,48 \pm 0,22$ & 1,09 & 4,64 & $23,03-23,93$ \\
\hline D. glabra & $18,55-21,18$ & $19,89 \pm 0,16$ & 0,80 & 4,04 & $19,56-20,23$ \\
\hline D. granatensis & $21,81-29,82$ & $24,63 \pm 0,45$ & 2,25 & 9,12 & $23,70-25,56$ \\
\hline D. nitens & $18,82-22,35$ & $20,46 \pm 0,17$ & 0,87 & 4,25 & $20,10-20,82$ \\
\hline D. occidentale & $21,46-25,38$ & $23,42 \pm 0,22$ & 1,11 & 4,73 & $22,97-23,88$ \\
\hline D. peruviana & $18,24-22,26$ & $19,90 \pm 0,23$ & 1,13 & 5,70 & $19,43-20,37$ \\
\hline D. cf. roraimensis & $21,67-26,11$ & $23,59 \pm 0,24$ & 1,21 & 5,12 & $23,09-24,09$ \\
\hline T. aspera & $23,36-29,42$ & $25,36 \pm 0,28$ & 1,41 & 5,55 & $24,78-25,94$ \\
\hline T. burserifolia & $21,58-29,32$ & $24,15 \pm 0,35$ & 1,77 & 7,33 & $23,42-24,88$ \\
\hline T. cf. lawrancei var. bolivianum & $20,45-26,65$ & $23,78 \pm 0,31$ & 1,57 & 6,60 & $23,13-24,43$ \\
\hline T. multiflora & $21,86-28,55$ & $25,76 \pm 0,35$ & 1,74 & 6,77 & $25,04-26,48$ \\
\hline \multirow[t]{2}{*}{ T. rhoifolia subsp. willdenowii } & $22,53-26,24$ & $24,30 \pm 0,24$ & 1,17 & 4,82 & $23,82-24,79$ \\
\hline & \multicolumn{5}{|c|}{ Diâmetro equatorial (VP) } \\
\hline D. chimantensis & $22,44-26,83$ & $24,32 \pm 023$ & 1,14 & 4,68 & $23,85-24,80$ \\
\hline D. glabra & $18,24-20,45$ & $19,28 \pm 0,11$ & 0,56 & 2,92 & $19,05-19,52$ \\
\hline D. granatensis & - & $24,50^{*}$ & - & - & - \\
\hline D. nitens & $18,46-21,86$ & $19,77 \pm 0,20$ & 1,02 & 5,16 & $19,36-20,18$ \\
\hline D. occidentale & $21,81-27,06$ & $23,99 \pm 0,27$ & 1,35 & 5,61 & $23,43-24,54$ \\
\hline D. peruviana & - & $18,49^{*}$ & - & - & - \\
\hline D. cf. roraimensis & - & $24,01^{*}$ & - & - & - \\
\hline T. aspera & - & $26,26^{*}$ & - & & - \\
\hline T. burserifolia & $20,95-27,15$ & $23,42 \pm 0,31$ & 1,54 & 6,60 & $22,78-24,06$ \\
\hline T. cf. lawrancei var. bolivianum & $20,14-25,70$ & $23,10 \pm 0,29$ & 1,47 & 6,36 & $22,49-23,71$ \\
\hline T. multiflora & $19,59-26,92$ & $23,93 \pm 0,35$ & 1,77 & 7,40 & $23,20-24,66$ \\
\hline T. rhoifolia subsp. willdenowii & $22,17-26,43$ & $24,19 \pm 0,22$ & 1,11 & 4,60 & $23,96-24,41$ \\
\hline
\end{tabular}

$*_{\mathrm{n}}<10$

pelo tamanho do pólen. Os dados disponíveis mostram que o gênero Trattinnickia é estenopolínico e a abertura revelou-se o único caráter que permitiu separar T. burserifolia (brevicolpada) das demais espécies estudadas que são longicolpadas.

Forman et al. (1989) estudaram quatro espécies de Dacryodes: D. expansa (Ridley) H. J. Lam, $D$. hexandra Griseb., D. kingii (Engl.) Kalkm. e $D$. rostrata (B1.) H. J. Lam, enquanto Segaar \& van der
Ham (1993) descreveram os grãos de pólen de $D$. costata (A. W. Bennett) H. J. Lam, D. letestui (Pellegrin) H. J. Lam e D. nervosa (H. J. Lam) Leenhouts, encontrando características polínicas similares quanto à forma prolata-esferoidal a subprolata, destacando a grande variabilidade na ornamentação da exina, descrita como estriado-reticulada, psilado-perfurada, psilada a verrugada, estriadorugulada, rugulada até perfurada parecendo sua- 
Tabela 3. Média aritmética das medidas das aberturas dos grãos de pólen de espécies de Dacryodes e Trattinnickia $(\mathrm{n}=10)$.

\begin{tabular}{|c|c|c|c|c|c|}
\hline \multirow{2}{*}{ Espécies } & \multicolumn{2}{|c|}{ Colpo $(\mu \mathrm{m})$} & \multicolumn{3}{|c|}{ Endoabertura $(\mu \mathrm{m})$} \\
\hline & Comprimento & Largura & Comprimento & Largura & Costa \\
\hline D. chimantensis & 20,03 & 1,81 & 6,16 & 9,45 & 1,04 \\
\hline D. glabra & 15,13 & 1,88 & 4,68 & 8,12 & 1,26 \\
\hline D. granatensis & 16,23 & 1,40 & 5,45 & 7,29 & 1,28 \\
\hline D. nitens & 15,94 & 1,30 & 4,44 & 7,39 & 1,00 \\
\hline D. occidentale & 10,28 & 1,83 & 6,58 & 9,33 & 2,06 \\
\hline D. peruviana & 22,02 & 1,30 & 4,20 & 7,18 & 1,42 \\
\hline D. cf. roraimensis & 19,48 & 1,82 & 6,06 & 8,67 & 1,13 \\
\hline T. aspera & 17,92 & 1,59 & 6,52 & 11,68 & 1,39 \\
\hline T. burserifolia & 14,57 & 1,75 & 5,10 & 8,30 & 1,26 \\
\hline T. cf. lawrancei var. bolivianum & 18,04 & 1,70 & 5,95 & 10,97 & 1,19 \\
\hline T. multiflora & 17,32 & 2,58 & 5,38 & 9,27 & 1,06 \\
\hline T. rhoifolia subsp. willdenowii & 20,32 & 1,90 & 6,07 & 11,37 & 1,15 \\
\hline
\end{tabular}

Tabela 4. Média aritmética das medidas da exina dos grãos de pólen de espécies de Dacryodes e Trattinnickia, em vista equatorial, nas regiões da endoabertura e do pólo $(\mathrm{n}=10)$.

\begin{tabular}{lcccc}
\hline & \multirow{2}{*}{$\begin{array}{c}\text { Endoaber- } \\
\text { tura }(\mu \mathrm{m})\end{array}$} & \multicolumn{3}{c}{ Pólo $(\mu \mathrm{m})$} \\
\cline { 3 - 5 } & Exina & Exina & Sexina & Nexina \\
\hline D. chimantensis & 3,52 & 1,89 & 0,81 & 1,15 \\
D. glabra & 3,40 & 2,22 & 1,11 & 1,12 \\
D. granatensis & 3,95 & 2,21 & 1,15 & 1,11 \\
D. nitens & 3,17 & 1,92 & 0,90 & 1,11 \\
D. occidentale & 3,71 & 2,38 & 0,90 & 1,46 \\
D. peruviana & 3,33 & 1,88 & 0,91 & 1,05 \\
D. cf. roraimensis & 3,60 & 1,86 & 0,83 & 1,09 \\
T. aspera & 4,62 & 2,03 & 0,93 & 1,15 \\
T. burserifolia & 4,03 & 1,97 & 0,96 & 1,09 \\
T. cf. lawrancei & & & & \\
var. bolivianum & 4,73 & 1,98 & 0,95 & 1,04 \\
T. multiflora & 4,27 & 1,96 & 0,91 & 1,08 \\
T. rhoifolia & & & & \\
subsp. willdenowii & 3,81 & 2,07 & 0,83 & 1,29 \\
\hline
\end{tabular}

vemente rugulada. Assim, com base na literatura, o gênero Dacryodes é euripolínico.

As espécies de Dacryodes aqui estudadas mostraram-se estenopolínicas com a exina psilada em microscopia óptica e psilado-perfurada ao MEV; D. glabra e D. nitens, com grãos de pólen pequenos, separam-se das outras espécies que apresentam grãos de pólen médios; e D. occidentale, com grãos de pólen brevicolpados, distingue-se das demais espécies, palinologicamente muito similares entre si.

Os dados polínicos disponíveis na literatura para os demais gêneros da tribo Canarieae mostram que esta é caracterizada por grãos de pólen médios, prolato-esferoidais, subprolatos a prolatos, (2)-3-(4)colporados, colpos longos a moderadamente longos, endoabertura lalongada com costa, exina psiladoperfurada, rugulada com ou sem espículos, estriada, estriado-reticulada, estriado-rugulada ou microrreticulada.

Para a família Burseraceae foram descritos dois tipos polínicos bem divergentes, pertencentes à tribo Canarieae. Um deles corresponde a Rosselia bracteata Forman cujos grãos de pólen foram caracterizados em Forman et al. (1994) pela presença da sexina reticulada, formada por muros altos, estreitos, simplescolumelados e nexina escabrada no fundo dos lúmens. De acordo com estes autores, esta ornamentação também é observada em espécies de várias famílias como Acanthaceae (Barleria L. e Ruellia L.), Araliaceae, Euphorbiaceae (Bridelia montana Wall., B. scleroneura Muell. e Phyllantus L.) e Rutaceae (Toxosiphon Baillon) que podem ser vistas como exemplos da evolução convergente na morfologia polínica.

Segundo Forman et al. (1994), embora os grãos de pólen de Rosselia bracteata sejam únicos na família e tenham endoaberturas diferentes, do ponto de vista da forma, do colpo reduzido e da ornamentação, estão próximos a Commiphora (Bursereae). Isto sugeriria sua afinidade com Bursereae contradizendo as claras evidências de sua afinidade com Canarieae, indicadas pelos caracteres morfológicos e anatômicos (Forman et al. 1994). Para responder a esta disparidade, Forman et al. (1994) levantaram a hipótese de que poderia ser um caso de evolução 
Tabela 5. Média aritmética das medidas dos grãos de pólen dos materiais de comparação de espécies de Dacryodes e Trattinnickia $(\mathrm{n}=10)$.

\begin{tabular}{|c|c|c|c|c|}
\hline \multirow{2}{*}{ Espécies } & \multicolumn{2}{|c|}{ Vista equatorial $(\mu \mathrm{m})$} & \multirow{2}{*}{$\begin{array}{c}\text { Vista polar } \\
\quad(\mu \mathrm{m}) \\
\text { Diâm. equatorial }\end{array}$} & \multirow{2}{*}{$\mathrm{P} / \mathrm{E}$} \\
\hline & Diâm. polar & Diârn. equatorial & & \\
\hline D. occidentale (Fonnegra et al. 3043) & 28,22 & 26,11 & 24,38 & 1,08 \\
\hline D. occidentale (Hernández et al. 559) & 26,22 & 23,08 & 21,41 & 1,14 \\
\hline D. occidentale (Monsalve 616) & 25,95 & 20,86 & 19,39 & 1,24 \\
\hline D. cf. peruviana (Monsalve 597) & 25,53 & 18,41 & - & 1,39 \\
\hline D. peruviana (Palacios 2807) & 30,06 & 27,16 & 26,82 & 1,11 \\
\hline D. peruviana (Prance et al. 16147) & 26,24 & 22,86 & 23,16 & 1,15 \\
\hline D. peruviana (Schunke 4445$)$ & 30,38 & 26,84 & 27,14 & 1,13 \\
\hline T. aspera (Ramírez \& Cárdenas 879) & 36,41 & 29,00 & - & 1,26 \\
\hline T. aspera (Cárdenas \& Ramírez 2723) & 37,33 & 27,53 & $26,93^{*}$ & 1,36 \\
\hline T. burserifolia (Coêlho \& Freitas 1765) & 29,90 & 23,47 & 22,77 & 1,27 \\
\hline T. burserifolia (Ducke s/n) & 29,01 & 24,80 & 23,79 & 1,17 \\
\hline T. burserifolia (Prance et al. 5828) & 29,46 & 25,15 & 25,57 & 1,17 \\
\hline T. burserifolia (Silva \& Maria 3330) & 29,24 & 22,16 & 22,38 & 1,32 \\
\hline T. burserifolia (Schulze 7262) & 29,97 & 24,43 & 23,41 & 1,23 \\
\hline T. burserifolia (Sperling et al. 6158) & 33,26 & 26,96 & 25,06 & 1,23 \\
\hline
\end{tabular}

$* \mathrm{n}=5$

divergente de um tipo ancestral, devido possivelmente a fatores ecológicos e/ou à polinização, visto que esta planta está isolada na ilha Rossel (Arquipélago Lousiade, Papua, Nova-Guiné).

O outro tipo polínico, ainda mais divergente do que este, já havia sido descrito para Scutinanthe brunnea Thw., caracterizado por grãos de pólen heteropolares, 3-colporados, onde cada abertura é bastante complexa, formada por um demicolpo curto, endoabertura subcircular com vestíbulo e cavidades laterais (Segaar \& van der Ham 1993).

Os dados da literatura mostram que, com exceção de Trattinnickia onde só foi observada exina psilado-perfurada, a ornamentação da exina varia em torno dos padrões rugulado, estriado, perfurado e microrreticulado, sendo em muitos casos difícil de precisar. Os resultados obtidos no presente estudo indicam que, na América do Sul, a tribo Canarieae é esteno-polínica, não tendo sido encontradas diferenças morfopolínicas significativas entre os grãos de pólen de Dacryodes e Trattinnickia.

\section{Referências bibliográficas}

AGUILAR-SIERRA, C.I. 1995. Contribuição à palino-taxonomia de Burseraceae. Tese de doutorado, Universidade de São Paulo, São Paulo.
CUATRECASAS, J. 1957. The american species of Dacryodes. Trop. Woods 106:46-65.

DALY, D.C. 1989. Studies in neotropical Burseraceae. II. Generic limits in New World Protieae and Canarieae. Brittonia 41:17-27.

ERDTMAN, G. 1952. Pollen morphology and plant taxonomy angiosperms. Almqvist \& Wiksell, Stockholm.

ERDTMAN, G. 1960. The acetolysis method. A revised description. Svensk Bot. Tidskr. 54:561-564.

FORMAN, L.L., BRANDHAM, P.E., HARLEY, M.M. \& LAWRENCE, T.J. 1989. Beiselia mexicana (Burseraceae) and its affinities. Kew Bull. 44:1-31.

FORMAN, L.L., VAN DER HAM, R.W.J.M., HARLEY, M.M \& LAWRENCE, T.J. 1994. Rosselia, a new genus of Burseraceae from the Lousiade Archipelago, Papua New Guinea. Kew Bull. 49:601-621.

MITRA, K., MONDAL, M. \& SAHA, S. 1977. The pollen morphology of Burseraceae. Grana 16:75-79.

ROUBIK, D.W. \& MORENO, J.E. 1991. Pollen and spores of Barro Colorado Island. Monogr. Syst. Bot. Missouri Bot. Gard. 36:73-74.

SEGAAR, P.J. \& VAN DER HAM, R.W.J.M. 1993. Pollen of Scutinanthe brunnea compared with other Burseraceous pollen types: a remarkable case of divergence. Rev. Palaeobot. Palynol. 79:297-334.

SOLEREDER, H. 1908. Systematic anatomy of the dicotyledons. Clarendon Press, Oxford.

SWART, J.J. 1942. A monograph of the genus Protium and some allied genera (Burseraceae). Rec. Trav. Bot. Néerl. 39:211-433. 